\title{
Existence of Mild solutions of Fractional order Hybrid Deferential Equations with Impulses
}

\author{
Prakashkumar H. Patel
}

Department of Mathematics, Faculty of Science, The M. S. University of Baroda, Vadodara, Gujarat, India

\section{ABSTRACT}

This article derive sufficient conditions for existence of mild solution for the hybrid fractional order differential equation with impulses of the form

$$
\begin{aligned}
{ }^{c} D^{\alpha}[x(t)-f(t, x(t))] & =g(t, x(t)) \\
\Delta x\left(t_{k}\right) & =I_{k} x\left(t_{k}\right) \\
x(0) & =x_{0}
\end{aligned}
$$

on a Banach space $X$ over interval $[0, T]$. The results are obtained using the concept of hybrid fixed point theorem. Finally an illustration is added to show validation of the derived results.

Keywords : Deferential Equations, Hybrid Dynamical System, Integro-Differential Equation

\section{INTRODUCTION}

Fractional calculus and differential equations gained lots of popularity from past few decades. This is because of its applications in all fields of science. For basic theory of fractional calculus and fractional differential equations one can refer the monographs [1, 2] and papers of [3-18]. Qualitative property like existence and uniqueness of mild solution for fractional order differential systems and integrodifferential systems using various techniques are found in articles [19-27]. Systems having jumps at fixed moments of time are modeled into impulsive differential equations. The study of existence and uniqueness of the solution for the impulsive differential equations and integro-differential equations with impulses are found in the papers of [28, 29].

On the other hand, the system having both the continuous and discrete behavior are modeled into hybrid systems. A system that can both flow (described by a differential equation) and jump (described by a state machine or automaton). Often, the term "hybrid dynamical system" is used, to distinguish over hybrid systems such as those that combine neural nets and fuzzy logic, or electrical and mechanical drivelines. A hybrid system has the benefit of encompassing a larger class of systems within its structure, allowing for more flexibility in modeling dynamic phenomena.

The system having both the continuous and discrete behavior are modeled into hybrid systems. The study of existence and uniqueness of fractional order systems are found in the articles [30-32].

In this article, we have derived sufficient conditions for the existence of mild solution of the hybrid system:

$$
\begin{aligned}
{ }^{c} D^{\alpha}[x(t)-f(t, x(t))] & =g(t, x(t)) \\
\Delta x\left(t_{k}\right) & =I_{k} x\left(t_{k}\right) \\
x(0) & =x_{0}
\end{aligned}
$$

over the interval $[0, T]$ on the partially ordered Banach space $X$. Here, $f, g:[0, T] \times X \rightarrow X$ are the functions satisfying the assumptions (3.1). For each $k=1,2, \cdots, p, I_{k}: X \rightarrow X$ is the jump operator applied at the fixed moment of time $t_{k}$. 


\section{Preliminaries}

Some basic definitions and properties of fractional calculus and fractional differential equations used in this article, are as follows:

Definition 2.1 ([28]) The Riemann-Liouville fractional integral operator of $\alpha>0$, of function $f \in L_{1}\left(\mathbb{R}_{+}\right)$is defined as

$$
\mathrm{I}_{\mathrm{t}_{0}+}^{\alpha} \mathrm{f}(\mathrm{t})=\frac{1}{\Gamma(\alpha)} \int_{\mathrm{t}_{0}}^{\mathrm{t}}(\mathrm{t}-\mathrm{s})^{\alpha-1} \mathrm{f}(\mathrm{s}) \mathrm{ds}
$$

provided the integral on right side exist. Where $\Gamma(\cdot)$ is gamma function.

Definition 2.2 ([29]) The Caputo fractional derivative of order $\alpha>0, n-1<\alpha<n, n \in \mathbb{N}$, is defined as

$$
{ }^{c} D_{t_{0}+}^{\alpha} f(t)=\frac{1}{\Gamma(n-\alpha)} \int_{t_{0}}^{t}(t-s)^{n-\alpha-1} \frac{d^{n} f(s)}{d s^{n}} d s,
$$

where the function $f(t)$ has absolutely continuous derivatives up to order $(n-1)$.

Fractional integral and differential operator satisfy following properties which is mentioned in Samko et. al. [1] and Kilbas et. al. [2].

Theorem 2.1 For $\alpha, \beta>0$ and $\mathrm{f}$ having absolutely continuous derivatives up to suitable order, then

(1) $\mathrm{I}_{\mathrm{t}_{0}+}^{\alpha} \mathrm{I}_{\mathrm{t}_{0}+}^{\beta} \mathrm{f}(\mathrm{t})=\mathrm{I}_{\mathrm{t}_{0}+}^{\alpha+\beta} \mathrm{f}(\mathrm{t})$

(2) $I_{t_{0}+}^{\alpha} I_{t_{0}+}^{\beta} f(t)=I_{t_{0}+}^{\beta} I_{t_{0}+}^{\alpha} f(t)$

(3) $I_{t_{0}+}^{\alpha}(f(t)+g(t))=I_{t_{0}+}^{\alpha} f(t)+I_{t_{0}+}^{\alpha} g(t)$

(4) $\mathrm{I}_{\mathrm{t}_{0}+}^{\alpha}{ }^{\mathrm{c}} \mathrm{D}_{\mathrm{t}_{0}+}^{\alpha} \mathrm{f}(\mathrm{t})=\mathrm{f}(\mathrm{t})-\mathrm{f}(0), \quad 0<\alpha<1$

(5) ${ }^{\mathrm{c}} \mathrm{D}_{\mathrm{t}_{0}+}^{\alpha} \mathrm{I}_{\mathrm{t}_{0}+}^{\alpha} \mathrm{f}(\mathrm{t})=\mathrm{f}(\mathrm{t})$

(6) ${ }^{\mathrm{c}} \mathrm{D}_{\mathrm{t}_{0}+}^{\alpha} \mathrm{f}(\mathrm{t})=\mathrm{I}_{\mathrm{t}_{0}+}^{1-\alpha} \mathrm{f}^{\prime}(\mathrm{t}), \quad 0<\alpha<1$

(7) ${ }^{\mathrm{c}} \mathrm{D}_{\mathrm{t}_{0}+}^{\alpha}{ }^{\mathrm{c}} \mathrm{D}_{\mathrm{t}_{0}+}^{\beta} \mathrm{f}(\mathrm{t}) \neq{ }^{\mathrm{c}} \mathrm{D}_{\mathrm{t}_{0}+}^{\alpha+\beta} \mathrm{f}(\mathrm{t})$

(8) ${ }^{c} D_{t_{0}+}^{\alpha}{ }^{c} D_{t_{0}+}^{\beta} f(t) \neq{ }^{c} D_{t_{0}+}^{\beta}{ }^{c} D_{t_{0}+}^{\alpha} f(t)$

For convenience, ${ }^{c} \mathrm{D}_{0+}^{\alpha}$ is taken as ${ }^{\mathrm{c}} \mathrm{D}^{\alpha}$.

\subsection{Notations}

- $\mathrm{X}=$ Banach space equipped with partial order.

- $\mathbb{R}_{+}=[0, \infty)$

- $\mathrm{C}([0, \mathrm{~T}], \mathrm{X})=\{\mathrm{x}:[0, \mathrm{~T}] \rightarrow \mathrm{X} / \mathrm{x}$ is continuous $\}$ with norm $\|\mathrm{x}\|=\sup _{\mathrm{t}}\|\mathrm{x}(\mathrm{t})\|$

- $\quad \operatorname{PC}([0, T], X)=\left\{x:[0, T] \rightarrow X / x\right.$ is partially continuous and has jump discontinuity at $\left.t=t_{k}\right\}$ with norm $\|x\|=\sup _{t}\|x(t)\|$

Definition 2.3 ([33]) An operator $\mathrm{T}: \mathrm{X} \rightarrow \mathrm{X}$ is called non-decreasing if the order relation preserved under $\mathrm{T}$, this means, for each $\mathrm{x}, \mathrm{y} \in \mathrm{X}$ such that $\mathrm{x} \leq \mathrm{y}$ implies $\mathrm{Tx} \leq \mathrm{Ty}$. 
Definition 2.4 ([33]) The order relation $\leq$ and a metric $d$ are compatible on nonempty set $X$ then convergence of sub sequence $\left\{x_{n_{k}}\right\}$ implies the convergence of $\left\{x_{n}\right\}$ for any monotone sequence $\left\{x_{n}\right\}$ in $X$.

Definition 2.5 ([33]) An upper semi-continuous and non-decreasing function $\psi: \mathbb{R}_{+} \rightarrow \mathbb{R}_{+}$is $\mathcal{D}$-function if $\psi(0)=0$

Definition 2.6 ([34]) An operator $\mathrm{T}: \mathrm{X} \rightarrow \mathrm{X}$ is called partially continuous at a $\in \mathrm{X}$ if for any $\epsilon>0$, there exist $\delta>$ 0 such that $\|\mathrm{Tx}-\mathrm{Ta}\|<\epsilon$ for all $\mathrm{x}$ comparable to a in $\mathrm{X}$ with $\|\mathrm{x}-\mathrm{a}\|<\delta$ and $\mathrm{T}$ is continuous on $\mathrm{X}$ if $\mathrm{T}$ is partially continuous at every a $\in \mathrm{X}$. In particular, if $\mathrm{T}$ is partially continuous on $\mathrm{X}$, then it is continuous on every chain $\mathrm{C}$ in $\mathrm{X}$. An operator T is called partially bounded if $\mathrm{T}(\mathrm{C})$ is bounded for every chain $\mathrm{C}$ in $\mathrm{X}$. An operator $\mathrm{T}$ is said to be uniformly partially bounded if all the chains $\mathrm{T}(\mathrm{C})$ in $\mathrm{X}$ are bounded.

Definition 2.7 ([34]) An operator $\mathrm{T}: \mathrm{X} \rightarrow \mathrm{X}$ is called partially compact if for any chain $\mathrm{C}$ in $\mathrm{X}$, the set $\mathrm{T}(\mathrm{C})$ are relatively compact subset of $\mathrm{X}$. An operator $\mathrm{T}$ is said to be partially totally bounded if for any totally ordered and bounded subset $\mathrm{C}$ of $\mathrm{X}$, the set $\mathrm{T}(\mathrm{C})$ is a relatively compact subset of $\mathrm{X}$. If $\mathrm{T}$ is partially continuous and partially totally bounded then $\mathrm{T}$ is partially completely continuous operator on $\mathrm{X}$.

Definition 2.8 ([34]) A mapping $\mathrm{T}: \mathrm{X} \rightarrow \mathrm{X}$ is partially nonlinear $\mathcal{D}$-Lipschitz if there is a $\mathcal{D}$-function $\psi: \mathbb{R}_{+} \rightarrow \mathbb{R}_{+}$ such that $\|\mathrm{Tx}-\mathrm{Ty}\| \leq \psi\|\mathrm{x}-\mathrm{y}\|$ for all compatible points $\mathrm{x}, \mathrm{y} \in \mathrm{X}$. If $\psi(\mathrm{r})<\mathrm{r}$ then $\mathrm{T}$ is called $\mathcal{D}$-contraction.

Theorem 2.2 ([34]) Let order and norm are compatible on a partially order Banach space. Let P and Q are nondecreasing operators on $\mathrm{X}$ such that:

(a) $\mathrm{P}$ is partially bounded nonlinear $\mathcal{D}$-contraction.

(b) $Q$ is partially continuous and partially compact.

(c) There exist an element $\mathrm{x}_{0} \in \mathrm{X}$ such that $\mathrm{x}_{0} \leq \mathrm{Px}_{0}+\mathrm{Qx}_{0}$. Then there exist a solution $\mathrm{x}^{*}$ in $\mathrm{X}$ of operator equation $\mathrm{Px}+\mathrm{Qx}=\mathrm{x}$. In addition, the sequence $\left\{\mathrm{x}_{\mathrm{n}}\right\}$ of successive iteration $\mathrm{x}_{\mathrm{n}+1}=\mathrm{Px}_{\mathrm{n}}+\mathrm{Qx}_{\mathrm{n}}$ converges monotonically to $x^{*}$.

\section{Main Results}

This section derived sufficient conditions for the mild solution for the equation (1.1).

Definition 3.1 Function $x(t) \in X$ is called mild solution of fractional order hybrid system (1.1) if $x(t)$ satisfies,

$$
x(t)= \begin{cases}x_{0}-f\left(t_{0}, x_{0}\right)+f(t, x(t))+\frac{1}{\Gamma(\alpha)} \int_{0}^{t}(t-s)^{\alpha-1} g(s, x(s)) d s, & t \in\left[0, t_{1}\right) \\ x\left(t_{k}^{+}\right)-f\left(t_{k}^{+}, x\left(t_{k}^{+}\right)\right)+f(t, x(t))+\frac{1}{\Gamma(\alpha)} \int_{t_{k}}^{t}(t-s)^{\alpha-1} g(s, x(s)) d s, & t \in\left[t_{k}, t_{k+1}\right) \\ x\left(t_{p}^{+}\right)-f\left(t_{p}^{+}, x\left(t_{p}^{+}\right)\right)+f(t, x(t))+\frac{1}{\Gamma(\alpha)} \int_{t_{p}}^{t}(t-s)^{\alpha-1} g(s, x(s)) d s, & t \in\left[t_{p}, T\right]\end{cases}
$$

for all value of $t \in[0, T]$.

We had made following assumption to discuss the existence results for the mild solution for the equation (1.1). 


\subsection{Assumptions}

- The functions $f:[0, T] \times X \rightarrow X$ and $g:[0, T] \times X \rightarrow X$ are non-decreasing and continuous.

- Function $f$ is $\mathcal{D}$-contraction with $\phi$ such that $\phi(r)<r$.

- There exist a positive constants $M_{f}$ and $M_{g}$ such that $|f(t, x)| \leq M_{f}$ and $|g(t, x)| \leq M_{g}$ respectively.

- The impulses $I_{k}: X \times X$ are continuous.

- There exist a function $u \in X$ such that $u$ is lower solution of the equation (1.1).

Theorem 3.1 (Existence Theorem) If assumptions (A1)-(A5) are satisfied then fractional order hybrid system (1.1) has mild solution in partially order Banach space $X$.

Proof. Over the interval $\left[t_{0}, t_{1}\right)$ the equation (1.1) becomes:

$$
{ }_{c} D^{\alpha[x(t)-f(t, x(t))]}=g(t, x(t))^{x(0)}=x 0
$$

We use theorem (2.2) to prove existence of mild solution of (3.2). Defining $P x(t)=f(t, x)$ and $Q=x_{0}-$ $f\left(0, x_{0}\right)+\frac{1}{\Gamma(\alpha)} \int_{0}^{t}(t-s)^{\alpha-1} g(s, x(s)) d s$ the equation (3.1) becomes $x=P x+Q x$. By theorem (2.2), this operator equation has a solution if conditions in hypotheses of the theorem (2.2) is satisfied.

\section{Step:1}

By conditions (A1), it is easily prove that the operators $P$ and $Q$ are non-decreasing. Also using (A2)-(A3), it can prove that $P$ is partially bounded nonlinear $\mathcal{D}$-contraction on $X$.

\section{Step:2}

In this step we proved that $Q$ is partially continuous and partially compact on $X$. Let, $\left\{x_{n}\right\}$ be a sequence in a chain $C$ in $X$ such that $x_{n} \rightarrow x$. Then,

$$
\left\|Q x_{n}(t)-Q x(t)\right\| \leq \frac{1}{\Gamma(\alpha)} \int_{0}^{t}(t-s)^{\alpha-1}\left\|g\left(s, x_{n}(s)\right)-g(s, x(s))\right\| d s
$$

Using continuity of $g$ and dominated convergence theorem, $\left\|Q x_{n}(t)-Q x(t)\right\| \rightarrow 0$ as $n \rightarrow \infty$ for all $t \in[0, T]$. Moreover the sequence $\left\{Q x_{n}\right\}$ is equi-continuous on $X$ as for, $t_{1}, t_{2} \in[0, T]$ with $t_{1}<t_{2}$,

$$
\begin{aligned}
& \left|Q x_{n}\left(t_{2}\right)-Q x_{n}\left(t_{1}\right)\right| \leq\left|\frac{1}{\Gamma(\alpha)} \int_{0}^{t_{1}}\left[\left(t_{2}-s\right)^{\alpha-1}-\left(t_{1}-s\right)^{\alpha-1}\right] g\left(s, x_{n}(s)\right) d s\right| \\
& +\frac{1}{\Gamma(\alpha)} \int_{t_{1}}^{t_{2}}\left(t_{2}-s\right)^{\alpha-1}\left|g\left(s, x_{n}(s)\right)\right| d s .
\end{aligned}
$$

By (A3), $\left|Q x_{n}\left(t_{2}\right)-Q x_{n}\left(t_{1}\right)\right| \rightarrow 0$ as $t_{2}-t_{1} \rightarrow 0$. Therefore, the sequence $\left\{Q x_{n}\right\}$ is equi-continuous. Thus $Q x_{n} \rightarrow Q x$ is uniformly on chain $C$ and hence, $Q$ is partially continuous on $X$.

\section{Step:3}

In this step we have shown that $Q$ is partially compact. Let $x \in C$ where $C$ is chain in $X$. Then,

$$
\|Q x(t)\| \leq\left\|x_{0}\right\|+\left\|f\left(0, x_{0}\right)\right\|++\frac{1}{\Gamma(\alpha)} \int_{0}^{t}(t-s)^{\alpha-1}\|g(s, x(s))\| d s
$$




$$
\leq M_{f}+\frac{M_{g}}{\alpha \Gamma(\alpha)}\left(t_{1}-t_{0}\right)^{\alpha}=K
$$

for all $t \in[0, T]$. Therefore $\|Q x(t)\| \leq K$ and hence $Q(C)$ is uniformly bounded.

Also,

$$
\begin{aligned}
& \left|Q x\left(t_{2}\right)-Q x\left(t_{1}\right)\right| \leq\left|\frac{1}{\Gamma(\alpha)} \int_{0}^{t_{1}}\left[\left(t_{2}-s\right)^{\alpha-1}-\left(t_{1}-s\right)^{\alpha-1}\right] g(s, x(s)) d s\right| \\
& +\frac{1}{\Gamma(\alpha)} \int_{t_{1}}^{t_{2}}\left(t_{2}-s\right)^{\alpha-1}|g(s, x(s))| d s
\end{aligned}
$$

Using (A3), $\left|Q x\left(t_{2}\right)-Q x\left(t_{1}\right)\right| \rightarrow 0$ as $t_{2} \rightarrow t_{1}$ uniformly for all $x \in C$. Therefore $Q(C)$ is relatively compact. Hence, $Q$ is partially compact.

Step-4:

By hypotheses (A5), the fractional hybrid system has lower solution $x(t)$ defined on $\left[0, t_{1}\right)$. That is

$$
\begin{aligned}
& { }^{c} D^{\alpha}[x(t)-f(t, x(t))]=g(t, x(t)) \\
& x(0) \leq x_{0} .
\end{aligned}
$$

Formulating mild solution, we can get

$$
x(t) \leq x_{0}-f\left(0, x_{0}\right)+f(t, x(t))+\frac{1}{\Gamma(\alpha)} \int_{0}^{t}(t-s)^{\alpha-1} g(s, u(s)) d s
$$

for all $t \in\left[0, t_{1}\right)$. Therefore $x \leq P x+Q x$.

Thus, from these steps we can concluded that the operators $P$ and $Q$ satisfy all the conditions of the hypotheses of the theorem (2.2) and hence the equation (3.2) has mild solution over the interval $\left[0, t_{1}\right)$. Moreover, The partially right limit of $x$ at $t=t_{1}$ exist and $x(t)$ is partially continuous at $t=0$.

Over the interval $\left[t_{1}, t_{2}\right)$ the equation becomes:

$$
{ }_{c} D^{\alpha[x(t)-f(t, x(t))]}=g(t, x(t)) x(t 1)=x(t 1+)=x(t 1)+I 1 x(t 1)
$$

Defining $P x(t)=f(t, x)$ and $Q=x\left(t_{1}\right)+I_{1} x\left(t_{1}\right)-f\left(t_{1}, x_{t_{1}}+I_{1} x\left(t_{1}\right)+\frac{1}{\Gamma(\alpha)} \int_{t_{1}}^{t}(t-s)^{\alpha-1} g(s, x(s)) d s\right.$ the equation (3.1) becomes $x=P x+Q x$. By theorem (2.2), this operator equation has solution if conditions in hypotheses of the theorem (2.2) is to be satisfied. By assuming (A1)-(A5) and following similar arguments it can easily show that the equation (3.3) has a mild solution over the interval $\left[t_{1}, t_{2}\right)$. Moreover $x(t)$ is partially left continuous at $t=t_{2}$ and the right limit at $t=t_{1}$ exist.

Continuing similar process it is shown that the equation

$$
{ }_{c} D^{\alpha[x(t)-f(t, x(t))]}=g(t, x(t)) x(t k)=x(t k+)=x(t k)+I k x(t k)
$$

has a mild solution over the interval $\left[t_{k}, t_{k+1}\right)$ which is partially left continuous and right limit exist for all $k=$ $1,2, \cdots, p$. Substituting $k=p$ in equation (3.4), it has mild solution over the interval $\left[t_{p}, T\right]$ which is partially left continuous and having right limit.

Hence, the equation (1.1) has mild solution which belongs to the set $P C([0, T], X)$. 


\subsection{Example}

Consider the following hybrid fractional differential equation:

$$
\begin{aligned}
& { }^{c} D^{\alpha}[x(t)-f(t, x(t))]=\frac{1}{2} \tan ^{-1} x(t) \\
& \Delta x\left(\frac{1}{2}\right)=\frac{1}{2} x\left(\frac{1}{2}\right) \\
& x(0)=1
\end{aligned}
$$

over the interval $[0,1]$. Where,

$$
f(t, x)= \begin{cases}\frac{x}{2(x+1)}, & x \geq 0 \\ 0, & x<0\end{cases}
$$

Clearly, both the function $f, g$ and $I_{1}$ are continuous over the interval $[0,1] \times X$. The function $f$ and $g$ are nondecreasing and bounded as $|f(t, x)| \leq \frac{1}{2}$ and $g|(t, x)| \leq \frac{1}{2}$. This verifies assumptions (A1), (A3) and (A4).

The function $f$ is $\mathcal{D}$-contraction as:

$$
\begin{aligned}
0<f(t, x)-f(t, y) & =\frac{1}{2}\left[\frac{x}{x+1}-\frac{y}{y+1}\right] \\
& \leq \frac{1}{2}\left[\frac{x-y+y}{x-y+y-1}-\frac{y}{x-y+y-1}\right] \\
& \leq \frac{1}{2}\left[\frac{x-y}{x-y+1}\right] \leq \frac{1}{2}|x-y| .
\end{aligned}
$$

So, (A2) is also satisfies. Finally, the function

$$
x(t)= \begin{cases}0.5, & t \in\left[0, \frac{1}{2}\right) \\ 1, & t \in\left[\frac{1}{2}, 1\right]\end{cases}
$$

is lower solution of the equation. Therefore, hypotheses (A5) is satisfied.

Since, all the assumptions (A1)-(A5) are satisfied. Therefore by our results the equation (??) has a mild solution.

\section{CONCLUSION}

In this article, we have discussed the sufficient conditions for the existence of the mild solution of the impulsive hybrid fractional order system on the general Banach space using the hybrid fixed point theorem (2.2).

\section{VI.REFERENCES}

[1] Samko S. G. Kilbas A. A, Marichev O. I, Fractional Integrals and Derivatives; Theory and Applications, Gorden and Breach, (1993).

[2] Kilbas A. A., Srivastava H. M., Trujillo J. J, Theory and Applications of Fractional
Differential Equations, Elsevier Science, (2006).

[3] M. Renardy, W. J. Hrusa, J. A. Nohel, Mathematical problems in viscoelasticity, Longman Scientific and technical, Newyork, (1987).

[4] J. H. He, Approximate analytical solution for seepage flow with fractional derivatives in porous media, Computer Methods in Applied Mechanics and Engineering, 167(1998), 57-68.

[5] A. M. A. El-Sayed, Fractional order wave equation, International Journal of Theoretical Physics, 35(1996),311-322.

[6] V. Gafiychuk, B. Datsan, V. Meleshko, Mathematical modeling of time fractional 
reaction-diffusion system, Journal of Computational and Applied Mathematics, 220(2008), 215-225.

[7] R. Metzler, J. Klafter, The restaurant at the end of random walk, the recent developments in description of anomalous transport by fractional dynamics, Journal of Physics A: A Mathematical and General, 37(2004), 161-208

[8] ] J. H. He,Some applications of nonlinear fractional differential equations and their approximations, Bulletin of Science and Technology, 15(1999), 86-90.

[9] K. Sayevand, M. Fardi, E. Moradi, F. Hemati Boroujeni, Convergence analysis of homotopy perturbation method for Volterra integrodifferential equations of fractional order, Alexandria Engineering Journal (2013) 52, 807-812.

[10] J. Prakash, M. Kothandapani, V. Bharathi, Numerical approximations of nonlinear fractional differential difference equations by using modified He-Laplace method, Alexandria Engineering Journal (2016) 55, 645-651.

[11] Mohammad Tamsir, Vineet K. Srivastava, Revisiting the approximate analytical solution of fractional order gas dynamics equation, Alexandria Engineering Journal (2016) 55, 867-874.

[12] Olaniyi Samuel Iyiola, Gbenga Olay, The fractional Rosenau-Hyman model and its approximate solutioninka Ojo, Okpala Mmaduabuchi, Alexandria Engineering Journal (2016) 55, 1655-1659.

[13] Jyotindra C. Prajapati, Krunal B. Kachhia, Shiv Prasad Kosta, Fractional calculus approach to study temperature distribution within a spinning satellite, Alexandria Engineering Journal (2016) 55, 2345-2350.

[14] Harendra Singh, A new numerical algorithm for fractional model of Bloch equation in nuclear magnetic resonance, Alexandria Engineering Journal (2016) 55, 2863-2869.
[15] Muhammad Saqib, Farhad Ali, Ilyas Khan, Nadeem Ahmad Sheikh, Syed Aftab Alam Jan, Samiulhaq, Exact solutions for free convection flow of generalized Jeffrey fluid: A CaputoFabrizio fractional model, Alexandria Engineering Journal (2017) (in press).

[16] Nauman Raza, M. Abdullah, Asma Rashid Butt, Aziz Ullah Awan, Ehsan Ul Haque, Flow of a second grade fluid with fractional derivatives due to a quadratic time dependent shear stress, Alexandria Engineering Journal (2017) (in press).

[17] Y. Cheng, G. Guozhu, On the solution of nonlinear fractional order differential equations, Nonlinear Analysis: Theory, Methods and Applications, 310(2005), 26-29.

[18] B. Bonilla, M. Rivero, L. Rodriguez-Germa, J. J. Trujillo, Fractional differential equations as alternative models to nonlinear differential equations, Applied Mathematics and Computation 187 (2007) 79-88.

[19] D. Delbosco, L. Rodino, Existence and uniqueness for nonlinear fractional differential equations, Journal of Mathematical Analysis and Applications, 204(1996), 609-625. ry, Methods and Applications, 310(2005), 26-29.

[20] M. M. El-Borai, Semigroups and some nonlinear fractional differential equations, Applied Mathematics and Computations, 149(2004), 823-831.

[21] L. Byszewski, Theorems about the existence and uniqueness of solutions of semi linear evolution non-local Cauchy problem, Journal of Mathematical Analysis and Applications, 162(1991), 494-505.

[22] K. Balachandran, J. J. Trujillo, The non-local Cauchy problem for nonlinear fractional integro-differential equations in Banach spaces, Nonlinear Analysis, 72(2010), 45874593.

[23] M. Benshohra, J. Henderson, S. K. Ntouyas, A. Quahab, Existence results for fractional order functional differential equations with infinite 
delay, Journal of Mathematical Analysis and Applications,338(2008),1340-1350.

[24] L. Mahto, S. Abbas, Existence and uniqueness of Caputo fractional differential equations, AIP Conf. Proc., 1479(2012), 896-899.

[25] A. Neamaty, M. Yadollahzadeh, R. Darzi, On fractional differential equation with complex order, Prog. Frac. Differ. Appl. 1(2015),223227.

[26] R. Ibrahim, A. Kilicman, F. Damag, Existence and uniqueness for a class of iterative fractional differential equations, 78(2015), 1-13.

[27] M. Matar, J. Trujillo, Existence of local solutions for differential equations with arbitary fractional order, Arab. J. Math. 5(2016), 215-224.

[28] K. Balachandran, S. Kiruthika, J. J. Trujillo, Existence results for fractional impulsive integro-differential equations in Banach spaces, Commun Nonlinear Sci Numer Simulat, 16(2011), 1970-1977.

[29] K. Balachandran, S. Kiruthika, J. J. Trujillo, On fractional impulsive equations of sobolev type with nonlocal condition in Banach spaces, Computer and Mathematics with Applications, 62(2011),1157-1165.

[30] D. Somjaiwang and P. S. Ngiamsunthorn, Existence and approximations of solutions to fractional order hybrid differential equations, Advances in Differential equations, 2016(2016) 1-13.

[31] M. Herzallah and D. Baleanu, On fractional order hybrid differential equations, Abstr. Appl. Anal., 2014(2014), Aricle ID-389386.

[32] H. R. Kataria and P. H. Patel, Existence and uniqueness of solutions of fractional order hybrid differential equations with nonlocal Conditions, international journal of scientific research in science, engineering and technology,4 (2018), 1-6.

[33] B. Dhage, S. Dhage, Hybrid fixed point theory ordered normed linear spaces and applications to fractional integral equations, Differ. Equ. Appl., 5(2013), 155-184.

[34] B. Dhage, Partially condensing mappings in partially order normed linear spaces and applications to functional integral equation, Tamkang J. Math., 45(2014), 397-426.

\section{Cite this article as :}

Prakashkumar H. Patel, "Existence of Mild solutions of Fractional order Hybrid Deferential Equations with Impulses", International Journal of Scientific Research in Science, Engineering and Technology (IJSRSET), Online ISSN : 2394-4099, Print ISSN : 2395-1990, Volume 6 Issue 2, pp. 718-725, March-April 2019. Available

doi : https://doi.org/10.32628/IJSRSET218375

Journal URL : https://ijsrset.com/IJSRSET218375 